\title{
Radial mode dependence of optical beam shifts
}

\author{
N. Hermosa, ${ }^{1, *}$ Andrea Aiello, ${ }^{2,3}$ and J.P. Woerdman ${ }^{1}$ \\ ${ }^{1}$ Huygens Laboratory, Leiden University, P.O. Box 9504, 2300 RA Leiden, The Netherlands \\ ${ }^{2}$ Max Planck Institute for the Science of Light, Günther-Scharowsky-Strasse 1/Bau 34, 91058 Erlangen, Germany \\ ${ }^{3}$ Institute of Optics, Information and Photonics, University Erlangen-Nürnberg, Staudtstrasse 7/B2, 91058 Erlangen, \\ Germany \\ *Corresponding author: hermosa@physics.leidenuniv.nl
}

Compiled April 19, 2022

It is known that orbital angular momentum (OAM) couples the Goos-Hänchen and Imbert-Fedorov shifts. Here, we present the first study of these shifts when the OAM-endowed $L G_{\ell, p}$ beams have higher-order radial mode index $(p>0)$. We show theoretically and experimentally that the angular shifts are enhanced by $p$ while the positional shifts are not. (C) 2022 Optical Society of America

OCIS codes: 240.3695, 260.5430.

A bounded beam of light may experience a displacement and/or a deflection with respect to the geometric optics prediction upon reflection $[1,2]$. These shifts are known as the Goos-Hänchen (GH) [3] and the Imbert-Fedorov (IF) $[4,5]$ shifts. A shift in the plane of incidence indicates that the beam underwent a GH shift while a shift that is perpendicular to the plane of incidence connotes an IF shift. Moreover, these shifts are further distinguished because of their positional and angular parts [1]. The positional shift is the displacement of the center point of reflection while an angular shift happens when the beam deflects and propagates with a slight deviation from the law of reflection. The angular shift is responsible for the increasing beam excursion from the geometric optics prediction with propagation. All these shifts are in the order 0.1-10 wavelengths for the positional shifts and micro- to milliradians for the angular shifts.

These shifts may occur simultaneously or separately. Their presence depends on the polarization of the incident beam [1-5], the index gradient seen by the beam [6, 8-10], the beam's divergence (opening angle of the beam) $[1,7,11]$ and, as emphasized recently, on the modal structure of the beam [12-16]. In works that involve modal structures, the Laguerre-Gaussian beam is used as the definitive beam because of its orbital angular momentum (OAM). The OAM of light couples the four beam shifts (the positional GH and IF shifts and their angular counterparts). First described in [12], we subsequently proposed a matrix which was validated with experiments, that neatly summarizes this mixing [13]. But since we considered only the case of a LG radial mode index that is zero $(p=0)$, that matrix is only explicitly dependent on the azimuthal mode index, $\ell$. The most important result of [13] is the measurement of the OAMinduced positional GH and IF and the OAM-affected angular GH and IF shifts for $\ell=-1,1$. We extended the measurement for $|\ell|>1$ in [17]. The shifts are found to be linearly enhanced by the OAM.

In this Letter, we answer the question to what degree the complete spatial mode of a beam affects these shifts.
We first do theoretical calculations and extend our previous matrix formulation to the case of $p>0$. Next we report experiments on shifts associated with higher-order mode Laguerre-Gaussian modes of different azimuthal and radial mode indices.

Laguerre-Gaussian modes are a set of solutions of the paraxial wave equation of the form given by

$$
\begin{aligned}
u_{p}^{\ell}(r, \phi)= & {\left[\frac{2 p !}{\pi(p+|\ell|)}\right]^{1 / 2} \exp (i \ell \phi) \exp \left(\frac{i}{2} \frac{r^{2}}{z-i L}\right) } \\
& \times \exp \left[-i(2 p+|\ell|+1) \arctan \left(\frac{z}{L}\right)\right] \\
& \times \frac{1}{w(z)} L_{p}^{\ell}\left(\frac{2 r^{2}}{w^{2}(z)}\right)\left(\frac{\sqrt{2} r}{w(z)}\right)^{|\ell|}
\end{aligned}
$$

where $x=r \cos \phi, y=r \sin \phi, w_{0}$ is the fundamental beam waist, $L=k_{0} w_{0}^{2} / 2$ is the Rayleigh length, $w(z)=w_{0} \sqrt{1+(z / L)^{2}}$ is the beam waist at $z, k_{0}$ is the center wave vector, and $L_{p}^{\ell}(x)$ denotes the generalized Laguerre polynomial of order $(\ell, p)$ with $p \geq 0$ and $\ell$ as integers. This beam is characterized by the indices $|\ell|$ and $p$, where $\ell$ is the azimuthal mode index and $p+1$ is the number of radial nodes [18]. There has been little interest in the index $p$; mainly for use in optical manipulation and trapping [19-22]. However, higher LG modes $(p,|\ell|>0)$ are increasingly being noticed, for instance in gravitational wave detection. As a matter of fact, some tabletop experiments are being done to test their suitability [23-25].

The theoretical derivation of the effect of the radial mode index follows from $[1,13]$. The incident and reflected beams are assumed to be Gaussian. The incident beam is then decomposed into plane wave components and the Fresnel reflection coefficients are applied to the $s$ and $p$ polarization components of the wave, respectively. The shift is obtained by summing all the reflected plane wave components, and taking the centroid of the intensity distribution. 
Following the notation in [13] and noting that we are using a median detector [26,27], we arrive at a matrix which is explicitly dependent on both $\ell$ and $p$, given by,

$$
\left[\begin{array}{c}
\Delta_{G H}^{\ell} \\
\Theta_{I F}^{\ell} \\
\Delta_{I F}^{\ell} \\
\Theta_{G H}^{\ell}
\end{array}\right]=\left[\begin{array}{cccc}
1 & -2 \ell & 0 & 0 \\
0 & \xi(\ell, p) & 0 & 0 \\
0 & 0 & 1 & 2 \ell \\
0 & 0 & 0 & \xi(\ell, p)
\end{array}\right]\left[\begin{array}{c}
\Delta_{G H}^{0} \\
\Theta_{I F}^{0} \\
\Delta_{I F}^{0} \\
\Theta_{G H}^{0}
\end{array}\right]
$$

where $\Delta_{G H}^{0}$ and $\Delta_{I F}^{0}$ are the dimensionless positional GH and IF shifts, respectively, and $\Theta_{G H}^{0}$ and $\Theta_{I F}^{0}$ are the dimensionless angular GH and IF shifts, respectively, for a $T E M_{00}$ beam. These are given by,

$$
\begin{gathered}
\Delta_{G H}^{0}=w_{p} \operatorname{Im}\left(\frac{\partial \ln r_{p}}{\partial \theta}\right)+w_{s} \operatorname{Im}\left(\frac{\partial \ln r_{s}}{\partial \theta}\right), \\
-\Theta_{G H}^{0}=w_{p} \operatorname{Re}\left(\frac{\partial \ln r_{p}}{\partial \theta}\right)+w_{s} \operatorname{Re}\left(\frac{\partial \ln r_{s}}{\partial \theta}\right), \\
\Delta_{I F}^{0}=-\frac{a_{p} a_{s} \cot \theta}{R_{p}^{2} a_{p}^{2}+R_{s}^{2} a_{s}^{2}} \times \\
{\left[\left(R_{p}^{2}+R_{s}^{2}\right) \sin \eta+2 R_{p} R_{s} \sin \left(\eta-\varphi_{p}+\varphi_{s}\right)\right],}
\end{gathered}
$$

$$
\Theta_{I F}^{0}=\frac{a_{p} a_{s} \cot \theta}{R_{p}^{2} a_{p}^{2}+R_{s}^{2} a_{s}^{2}}\left[\left(R_{p}^{2}-R_{s}^{2}\right) \cos \eta\right]
$$

with $w_{s / p}=R_{s / p}^{2} a_{s / p}^{2} /\left(R_{p}^{2} a_{p}^{2}+R_{s}^{2} a_{s}^{2}\right)$, and $r_{s / p}=$ $R_{s / p} \exp \left(i \varphi_{s / p}\right)$ the Fresnel reflection coefficient evaluated at the incident angle $\theta_{i}$ and $a_{s / p}$ the electric field components. The shifts $x$ and $y$ are the sum of two contributions and are given by $k_{0} x=\Delta_{G H}^{\ell}+(z / L) \Theta_{G H}^{\ell}$ and $k_{0} y=\Delta_{I F}^{\ell}+(z / L) \Theta_{I F}^{\ell}$, respectively.

Finally, the matrix element $\xi(\ell, p)$ calculated for a median detector is $\xi(\ell, p)=2 I_{+} / I_{-}$where

$$
\begin{aligned}
I_{ \pm}(\ell, p)= & \sum_{a=0}^{p} \sum_{b=0}^{p} \frac{(-1)^{a+b}}{a ! b !}\left(\begin{array}{c}
p+|\ell| \\
p-a
\end{array}\right)\left(\begin{array}{c}
p+|\ell| \\
p-b
\end{array}\right) \\
& \times \Gamma\left(a+b+|\ell|+1 \pm \frac{1}{2}\right) .
\end{aligned}
$$

It follows from (2) that the positional shift is not affected by the $p$ index. Only the angular shift components are influenced by it. Moreover, the matrix elements $M_{22}$ and $M_{44}$ are the same. This means that the enhancements of the angular GH and IF shifts are the same.

Our experimental setup is shown in Fig. 1. We use an open cavity HeNe laser $(\lambda=632.8 \mathrm{~nm})$ and placed two crossed copper wires $(d \sim 90 \mu m)$ inside the cavity. The wires force the cavity to emit a higher-order Hermite-Gaussian mode $\left(H G_{m, n}\right)$. The integer mode indices $m$ and $n$ can be dialed by properly shifting the

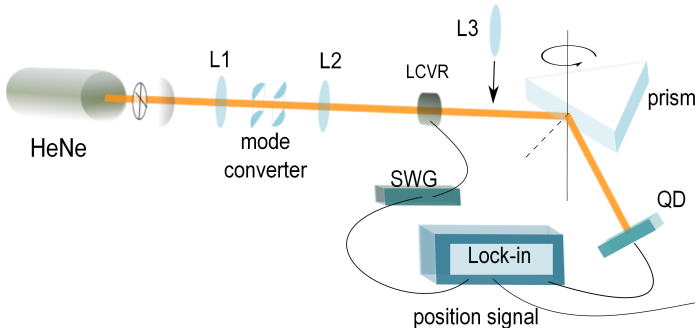

Figure 1. (color online) The experimental set-up used to measure the polarization-differential beam shifts as a function of the incident angle. LCVR is the variable retarder. SWG is the square wave generator. QD is the quadrant detector. L1, L2, and L3 are lenses. More details are in the text.

intracavity wires. With an astigmatic mode converter, the $H G_{m, n}$ modes are then converted into $L G_{\ell, p}$ modes with transformations $\ell=m-n$ and $p=\min (m, n)$ as described in $[28,29]$. L1 is the mode matching lens while lens L2 collimates the beam. The beam then passes through an LC variable retarder (LVCR) which sets the beam's polarization state. The LCVR (Meadowlark) is driven by a square wave generator at $2.5 \mathrm{~Hz}$. This wave generator also provides the reference wave of the lock-in amplifier (EG\&G 5210). The positional shifts are measured with a collimated beam while the angular shifts are measured with a beam focused by lens L3 $(f=70 \mathrm{~mm})$. The displacement due to the angular shifts increases with the opening angle of the beam $\theta_{0}$, as $\theta_{0}^{2} / 2$ where $\theta_{0}=2 /\left(k_{0} \omega_{0}\right)[11,30]$.

The polarization differential shifts are measured between $45^{0} /-45^{0}$ polarization states. These states are specifically chosen because: 1) the dimensionless angular IF shift $\Theta_{I F}^{0}$ that is responsible for the $\Delta_{G H}^{\ell}$ and $\Theta_{I F}^{\ell}$ is maximum at these states; and 2) the contribution of $\Delta_{G H}^{0}, \Delta_{I F}^{0}$, and $\Theta_{G H}^{0}$ either have values equivalent to zero or cancel to zero upon measurement of the differential shift. These enable us to isolate the matrix elements $M_{12}$ and $M_{22}$ in (2).

After the polarization conditioning, the beam is made to reflect at different incident angles $\theta_{i}$ on a BK7 prism $(n=1.51)$ mounted on a $\theta-2 \theta$ rotation stage. The position of the reflected beam is measured by a quadrant detector (QD) whose signal is fed to the lock-in amplifier. These are done for different combinations of $\ell$ and $p$.

Figure 2 shows the measured matrix element $M_{12}$ as function of $\ell$ and with different values of $p$. The measured values collapse to the $M_{12}=-2 \ell$ line. This is correctly predicted by our theory. The matrix element changes linearly on $\ell$ but is independent on $p$. We have experimentally verified that the positional GH shift is not affected by the radial mode index (not shown).

The measured $M_{22}$ is shown in Fig. 3. The theoretical predictions are calculated from $\xi(\ell, p)$ for different $\ell$ and $p$ values. For $p>0$ we obtained greater enhancements. This confirms our theoretical prediction of the effect of 


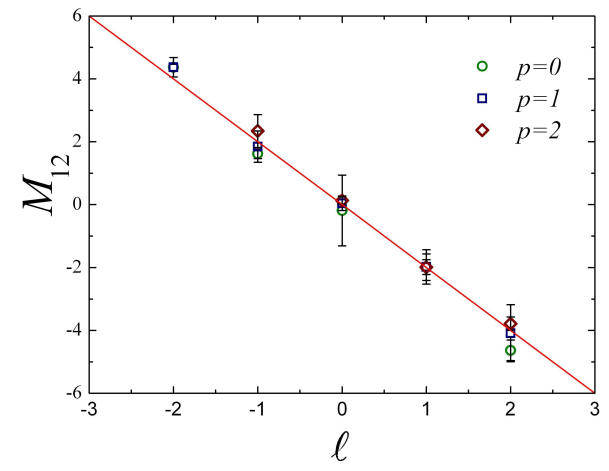

Figure 2. (color online) Matrix element for different values of $\ell$ and $p$. The solid line is $M_{12}=-2 \ell$.

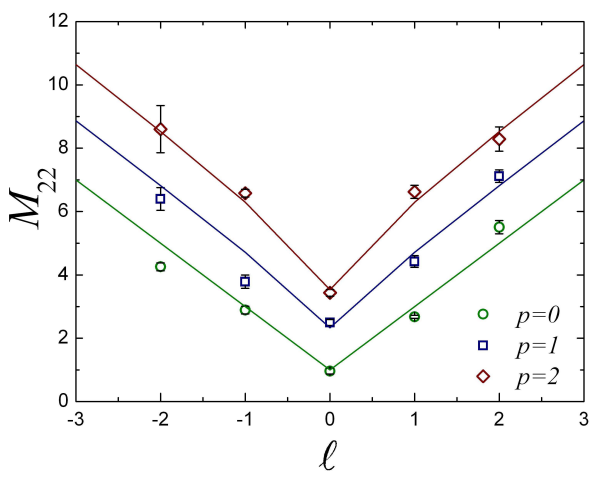

Figure 3. (color online) Matrix element $M_{22}$ for different values of $\ell$ and $p$. The solid line is $\xi(\ell, p)$. A discussion of $\xi(\ell, p)$ is in the text.

$p$ on the angular shifts. At $p=0$, the angular IF shift linearly increases as $1+2|\ell|$. Furthermore, had we used a centroid detector the increase of the angular shift would have been $\xi(\ell, p)=2 p+|\ell|+1$, a linear increase with $p$.

In conclusion, we have theoretically described and experimentally measured the positional GH shift and angular IF shifts for higher-order $L G_{\ell, p}$ to determine the role of mode indices $\ell$ and $p$. Specifically, we have observed that only the azimuthal index $\ell$ affects the positional shift while the angular shifts are enhanced by both the radial $p$ and the azimuthal $\ell$ indices. Higherorder $L G_{\ell, p}$ beams form a complete basis set for paraxial light beams contrary to $L G_{\ell, p=0}$ [18]. Hence with proper mode decomposition, beam shifts associated with arbitrary modes of light can be predicted based on our theoretical and experimental results.

This work is supported by the Foundation for Fundamental Research of Matter (FOM) and the European Union within FET Open-FP7 ICT as part of STREP Program 255914 Phorbitech.

\section{References}

1. A. Aiello and J. P. Woerdman, Opt. Lett. 33, 1437-1439 (2008). (document)

2. C.-F. Li, Phys. Rev. A 76, 15 (2006). (document)
3. F. Goos and H. Hanchen, Ann Phys 436, 1-11 (1947). (document)

4. F. I. Fedorov, Dokl.Akad. Nauk SSR 105, 465 (1955). (document)

5. C. Imbert, Phys. Rev. D 5, 787-796 (1972). (document)

6. M. Merano, A. Aiello, G. W. 't Hooft, M. P. Van Exter, E. R. Eliel, and J. P. Woerdman, Opt. Express 15, 15928-15934 (2007). (document)

7. A. Aiello, M. Merano, and J. P. Woerdman, Phys. Rev. A 80, 4 (2009). (document)

8. N. Hermosa, A. M. Nugrowati, A. Aiello, and J. P. Woerdman, Opt. Lett. 36, 3200-3202 (2011). (document)

9. J.-M. Ménard, A. Mattacchione, H. Van Driel, C. Hautmann, and M. Betz, Phys. Rev. B 82, 1-10 (2010). (document)

10. P. R. Berman, Phys. Rev. E 66 (2002). (document)

11. M. Merano, A. Aiello, M. P. Van Exter, and J. P. Woerdman, Nat Photonics 3, 337-340 (2009). (document)

12. K. Y. Bliokh, I. V. Shadrivov, and Y. S. Kivshar, Opt. Lett. 34, 389-391 (2009). (document)

13. M. Merano, N. Hermosa, J. Woerdman, and A. Aiello, Phys. Rev. A 82, 023817(2010). (document)

14. A. Aiello and J. Woerdman, Opt. Lett. 36, 543-545 (2011). (document)

15. R. Dasgupta and P. Gupta, Opt. Commun. 257, 91-96 (2006). (document)

16. D. Golla and S.D. Gupta, Pramana J Phys 76, 603-612 (2011). (document)

17. N. Hermosa, M. Merano, A. Aiello, and J. P. Woerdman, in Proc. of SPIE 7950, 79500F-79500F-7 (2011). (document)

18. A. Siegman, Lasers, (University Science Books, Herndon, VA, 1986). (document)

19. S. Kennedy, M. Szabo, H. Teslow, J. Porterfield, and E. Abraham, Phys. Rev. A 66, 1-5 (2002). (document)

20. N. Matsumoto, T. Ando, T. Inoue, Y. Ohtake, N. Fukuchi, and T. Hara, J. Opt. Soc. Am. A 25, 16421651 (2008). (document)

21. V. Garces-Chavez, D. McGloin, M. J. Padgett, W. Dultz, H. Schmitzer, and K. Dholakia, Phys. Rev. Lett. 91, 093602 (2003). (document)

22. J. Tempere, J. T. Devreese, and E. R. I. Abraham, Phys. Rev. A 64, 23603 (2001). (document)

23. S. Chelkowski, S. Hild, and A. Freise, Phys. Rev. D 79, 12 (2009). (document)

24. P. Fulda, K. Kokeyama, S. Chelkowski, and A. Freise, Phys. Rev. D 82, 1-6 (2010). (document)

25. M. Granata, C. Buy, R. Ward, and M. Barsuglia, Phys. Rev. Lett. 105, 231102 (2010). (document)

26. N. Hermosa, A. Aiello, and J. P. Woerdman, Opt. Lett. 36, 409-11(2011). (document)

27. If a centroid detector is used instead of a median detector, $2 \ell \longrightarrow \ell$ and $\xi(\ell, p)=2 p+|\ell|+1$. (document)

28. M. W. Beijersbergen, L. Allen, H.E.L.O. van der Veen, and J. P. Woerdman, Opt. Communications 96, 123-132, (1993). (document)

29. L. Allen, M. Beijersbergen, R. Spreeuw, and J. Woerdman, Phys. Rev. A 45, 8185-8189 (1992). (document)

30. M. Merano, N. Hermosa, A. Aiello, and J. P. Woerdman, Opt. Lett. 35, 3562-3564 (2010). (document) 


\section{Informational Fourth Page}

\section{References}

1. A. Aiello and J. P. Woerdman, "Role of beam propagation in Goos-Hänchen and Imbert-Fedorov shifts," Opt. Lett. 33, 1437-1439 (2008).

2. C.-F. Li, "Unified theory for Goos-Hänchen and ImbertFedorov effects," Phys. Rev. A 76, 15 (2006).

3. F. Goos and H. Hanchen, "Ein neuer und fundamentaler Versuch zur Totalreflexion," Ann Phys 436, 1-11 (1947).

4. F. I. Fedorov, "K teorii polnogo otrazheniya," Dokl.Akad. Nauk SSR 105, 465 (1955).

5. C. Imbert, "Calculation and Experimental Proof of the Transverse Shift Induced by Total Internal Reflection of a Circularly Polarized Light Beam," Phys. Rev. D 5, 787-796 (1972).

6. M. Merano, A. Aiello, G. W. 't Hooft, M. P. Van Exter, E. R. Eliel, and J. P. Woerdman, "Observation of GoosHänchen shifts in metallic reflection.," Opt. Express 15, 15928-15934 (2007).

7. A. Aiello, M. Merano, and J. P. Woerdman, "Duality Between Spatial and Angular Shift in Optical Reflection," Phys. Rev. A 80, 4 (2009).

8. N. Hermosa, A. M. Nugrowati, A. Aiello, and J. P. Woerdman, "Spin Hall effect of light in metallic reflection," Opt. Lett. 36, 3200-3202 (2011).

9. J.-M. Ménard, A. Mattacchione, H. Van Driel, C. Hautmann, and M. Betz, "Ultrafast optical imaging of the spin Hall effect of light in semiconductors," Phys. Rev. B 82, 1-10 (2010).

10. P. R. Berman, "Goos-Hanchen shift in negatively refractive media," Phys. Rev. E 66 (2002).

11. M. Merano, A. Aiello, M. P. Van Exter, and J. P. Woerdman, "Observing angular deviations in the specular reflection of a light beam," Nat Photonics 3, 337-340 (2009).

12. K. Y. Bliokh, I. V. Shadrivov, and Y. S. Kivshar, "GoosHänchen and Imbert-Fedorov shifts of polarized vortex beams.," Opt. Lett. 34, 389-391 (2009).

13. M. Merano, N. Hermosa, J. Woerdman, and A. Aiello, "How orbital angular momentum affects beam shifts in optical reflection," Phys. Rev. A 82, 023817(2010).

14. A. Aiello and J. Woerdman, "Goos-Hänchen and Imbert-Fedorov shifts of a nondiffracting Bessel beam," Opt. Lett. 36, 543-545 (2011).

15. R. Dasgupta and P. Gupta, "Experimental observation of spin-independent transverse shift of the centre of gravity of a reflected Laguerre-Gaussian light beam," Opt. Commun. 257, 91-96 (2006).

16. D. Golla and S.D. Gupta, "Goos-Hänchen shift for higher-order Hermite-Gaussian beams," Pramana J Phys 76, 603-612 (2011).

17. N. Hermosa, M. Merano, A. Aiello, and J. P. Woerdman, "Orbital angular momentum induced beam shifts," in Proc. of SPIE 7950, 79500F-79500F-7 (2011).

18. A. Siegman, Lasers, (University Science Books, Herndon, VA, 1986).

19. S. Kennedy, M. Szabo, H. Teslow, J. Porterfield, and E. Abraham, "Creation of Laguerre-Gaussian laser modes using diffractive optics," Phys. Rev. A 66, 1-5 (2002).

20. N. Matsumoto, T. Ando, T. Inoue, Y. Ohtake, N.
Fukuchi, and T. Hara, "Generation of high-quality higher-order Laguerre-Gaussian beams using liquidcrystal-on-silicon spatial light modulators," J. Opt. Soc. Am. A 25, 1642-1651 (2008).

21. V. Garces-Chavez, D. McGloin, M. J. Padgett, W. Dultz, H. Schmitzer, and K. Dholakia, "Observation of the transfer of the local angular momentum density of a multiringed light beam to an optically trapped particle," Phys. Rev. Lett. 91, 093602 (2003).

22. J. Tempere, J. T. Devreese, and E. R. I. Abraham, "Vortices in Bose-Einstein condensates confined in a multiply connected Laguerre-Gaussian optical trap," Phys. Rev. A 64, 23603 (2001).

23. S. Chelkowski, S. Hild, and A. Freise, "Prospects of higher-order Laguerre-Gauss modes in future gravitational wave detectors," Phys. Rev. D 79, 12 (2009).

24. P. Fulda, K. Kokeyama, S. Chelkowski, and A. Freise, "Experimental demonstration of higher-order LaguerreGauss mode interferometry," Phys. Rev. D 82, 1-6 (2010).

25. M. Granata, C. Buy, R. Ward, and M. Barsuglia, "Higher-order Laguerre-Gauss mode generation and interferometry for gravitational wave detectors," Phys. Rev. Lett. 105, 231102 (2010).

26. N. Hermosa, A. Aiello, and J. P. Woerdman, "Quadrant detector calibration for vortex beams," Opt. Lett. 36, 409-11(2011).

27. If a centroid detector is used instead of a median detector, $2 \ell \longrightarrow \ell$ and $\xi(\ell, p)=2 p+|\ell|+1$.

28. M. W. Beijersbergen, L. Allen, H.E.L.O. van der Veen, and J. P. Woerdman"Astigmatic laser mode converters and transfer of orbital angular momentum," Opt. Communications 96, 123-132, (1993).

29. L. Allen, M. Beijersbergen, R. Spreeuw, and J. Woerdman, "Orbital angular momentum of light and the transformation of Laguerre-Gaussian laser modes," Phys. Rev. A 45, 8185-8189 (1992).

30. M. Merano, N. Hermosa, A. Aiello, and J. P. Woerdman, "Demonstration of a quasi-scalar angular Goos-Hänchen effect," Opt. Lett. 35, 3562-3564 (2010). 